\title{
Evaluation of an African swine fever (ASF) vaccine strategy incorporating priming with an alphavirus-expressed antigen followed by boosting with attenuated ASF virus
}

\author{
Maria V. Murgia ${ }^{1} \cdot$ Mark Mogler $^{2} \cdot$ Andrea Certoma $^{3} \cdot$ Diane Green $^{3} \cdot$ Paul Monaghan $^{3} \cdot$ David T. Williams $^{3} \cdot$ \\ Raymond R. R. Rowland ${ }^{1} \cdot$ Natasha N. Gaudreault $^{1}$ (i)
}

Received: 14 December 2017 / Accepted: 30 September 2018 / Published online: 26 October 2018

(c) Springer-Verlag GmbH Austria, part of Springer Nature 2018

\begin{abstract}
In this study, an alphavirus vector platform was used to deliver replicon particles (RPs) expressing African swine fever virus (ASFV) antigens to swine. Alphavirus RPs expressing ASFV p30 (RP-30), p54 (RP-54) or pHA-72 (RP-sHA-p72) antigens were constructed and tested for expression in Vero cells and for immunogenicity in pigs. RP-30 showed the highest expression in Vero cells and was the most immunogenic in pigs, followed by RP-54 and RP-sHA-p72. Pigs primed with two doses of the RP-30 construct were then boosted with a naturally attenuated ASFV isolate, OURT88/3. Mapping of p30 identified an immunodominant region within the amino acid residues 111-130. However, the principal effect of the primeboost was enhanced recognition of an epitope covered by the peptide sequence 61-110. The results suggest that a strategy incorporating priming with a vector-expressed antigen followed by boosting with an attenuated live virus may broaden the recognition of ASFV epitopes.
\end{abstract}

\section{Introduction}

African swine fever virus (ASFV), the only member of the family Asfarviridae [1], is responsible for a devastating disease in swine for which no commercial vaccines are available. The enveloped virus possesses a 170- to $190-\mathrm{kb}$ double-stranded DNA genome encoding more than 100 polypeptides [1-3]. Twelve viral proteins have been identified as highly immunogenic, including three structural proteins, p30, p54, p72, which are often combined in multivalent vaccine formulations [4]. Although live attenuated virus vaccines generally provide the greatest level of protection against ASF, subunit vaccines provide a safer alternative.

Handling Editor: Diego G. Diel.

Natasha N. Gaudreault

nng5757@ vet.k-state.edu

1 Diagnostic Medicine and Pathobiology, College

of Veterinary Medicine, Kansas State University, 1800

Denison Avenue, Manhattan, KS 66506, USA

2 Merck Animal Health, 1102 Southern Hills Drive Ste.101, Ames, IA 50010, USA

3 CSIRO Australian Animal Health Laboratory, 5 Portarlington Road, Geelong, VIC 3220, Australia
However, the development of an effective subunit vaccine against ASF has been largely unsuccessful. For example, subunit formulations consisting of baculovirus-expressed p54, p30 and p72 showed only modest improvements in reducing clinical signs and viremia [5-7]. DNA vaccines have the added advantage of targeting both humoral and cellmediated immunity. Pigs vaccinated with a plasmid expressing the extracellular domain of the ASFV hemagglutinin protein (HA) inserted upstream of a $554 \mathrm{p} 30$ fusion protein elicited good humoral and cellular immune responses [8]. However, pigs were not protected from virulent ASFV challenge, although the addition of ubiquitin to recruit CD8 cells did result in increased survival of challenged pigs [8]. Another approach for stimulating B- and T-cell responses is the incorporation of defective viruses for antigen delivery. Argilaguet et al. utilized an ASFV HA-p54p30 fusion protein cloned into a BacMam vector to immunize pigs. Even though no antibody response was detected, four out of six pigs survived challenge and showed no evidence of viremia [9]. One conclusion regarding the large body of work on ASF vaccines is the requirement for effective stimulation of both $\mathrm{B}$ and $\mathrm{T}$ cells along with the incorporation of multiple viral antigens.

A vectored vaccine that stimulates cellular immunity and expresses key antigens combined with an attenuated live 
virus could be a means to expand protection by allowing the opportunity to incorporate antigens of currently circulating ASFV genotypes or serotypes. Therefore, we sought to evaluate a prime-boost approach that incorporates targetedantigen delivery via defective alphavirus replicon particles (RPs) and an attenuated live ASFV strain. The alphavirus vaccine (alphavaccine) platform consists of defective single-cycle RPs derived from Venezuelan equine encephalitis virus (VEEV). For use as a vaccine, the structural protein genes from the VEEV genome are substituted with the antigen cDNA of interest and then co-electroporated into Vero cells together with two helper RNAs, which provide the VEEV capsid and envelope proteins in trans.

The alphavirus platform offers several advantages. First, the RPs specifically target antigen-presenting dendritic cells. Second, vaccination results in a single cycle of replication. Finally, the induction of anti-vector immunity is not known to interfere with subsequent vaccination. Furthermore, alphavaccines have been used safely in swine. Specifically, the alphavaccine platform has been successfully used for the delivery of GP5/M heterodimer of porcine reproductive and respiratory syndrome virus [unpublished data, 10, $11]$, the HA antigen of swine influenza virus [12, 13], and a truncation of the porcine epidemic diarrhea virus spike protein, which was conditionally licensed in the USA in 2013 [unpublished data, 14]. However, use of the alphavirus platform for ASF has not been explored. Therefore the first objective of this work was to test the expression of ASFV antigens by alphavirus RPs, and subsequently, their immunogenicity in pigs.

The second objective was to evaluate the effect of priming pigs with alphavirus RPs followed by boost with an attenuated live virus. The ASFV OURT88/3 strain is a naturally attenuated genotype I virus originally isolated from $O$. erraticus ticks [15]. OURT $88 / 3$ is considered a potential attenuated live virus vaccine candidate, as it confers good levels of protection in most animals challenged with virulent strains of a similar genotype $[16,17]$. However, occasional adverse side effects have been reported, which have hindered its development as a viable vaccine $[16,18]$. The purpose of the second objective was to address whether priming pigs with the alphavaccine interferes with replication of OURT $88 / 3$ and ultimately the serologic response of the vaccinated pigs.

Here, we describe the expression and immunogenicity of ASFV p30, p54 and HA modified p72 (HA-p72) antigens by alphavirus RPs. The p30 construct (RP-30), which was found to be expressed at high levels and was immunogenic in pigs, was selected for further testing with OURT88/3 in a prime-boost vaccination approach. The focus of our analysis was to characterize the effect of this vaccination strategy on the antigen-specific antibody response, which is critical for the development of an effective vaccine. Mapping of B cell epitopes showed that the combination of RP-30 and OURT88/3 resulted in broader recognition of p30 epitopes. This study serves a proof of concept study that presents a novel approach to potentially expand immunogenic epitopes.

\section{Materials and methods}

\section{Preparation of ASFV alphavirus constructs}

Nucleic acid sequences for $\mathrm{p} 30$, p54, and $\mathrm{p} 72$ from ASFV genotype I strain BA71V were obtained from GenBank (accession no. NP_042786, NP_042818, and NP_042775, respectively). A second $\mathrm{p} 72$ construct was prepared by adding the 17-amino-acid signal peptide and the first 180-amino-acid extracellular domain of ASFV HA from the E75 strain (GenBank accession no. FN557520.1; [8]). Sequences were codon-optimized for expression in mammalian cells, and a $6 \mathrm{xHis}$ tag added to the $\mathrm{C}$-terminal end of each protein. Constructs were synthesized de novo (DNA2.0, Menlo Park, CA) and cloned into the alphavirus replicon plasmid vector [19]. The resulting plasmids, pVEK-p30, pVEK-p54, pVEK-p72, and pVEK-sHA-p72, were transcribed in vitro and introduced into Vero cells by transfection along with RNA from VEEV capsid and glycoprotein helper RNAs. RP yield was quantified by titration on Vero cells [19].

For immunoblot detection of His-tagged ASFV proteins, infected Vero cells were lysed directly with radioimmunoprecipitation assay buffer (Thermo Fisher Scientific Inc., Pittsburgh, PA), incubated on ice for $20 \mathrm{~min}$, and then centrifuged at $5000 \times g$ for $15 \mathrm{~min}$. The clarified lysate was mixed $(1 / 1 ; \mathrm{v} / \mathrm{v})$ with $4 \mathrm{X}$ lithium dodecyl sulfate loading buffer and reducing agent (Invitrogen, Grand Island, NY) and boiled for $10 \mathrm{~min}$. Samples were loaded onto precast $12 \%$ bis-Tris polyacrylamide gels (Invitrogen, Grand Island, $\mathrm{NY}$ ) and electrophoresed in 2-(N-morpholino) ethanesulfonic acid buffer at $200 \mathrm{~V}$ for $90 \mathrm{~min}$, then transferred to a polyvinylidene difluoride (PVDF) membrane using an Invitrogen SureLock XL system at 0.18 amperes for $90 \mathrm{~min}$. Membranes were blocked with 5\% non-fat dry milk (NFDM) in Tris-buffered saline with $0.05 \%$ Tween-20 (TBST) for $1 \mathrm{~h}$ at room temperature, then for $1 \mathrm{~h}$ with anti-His $\mathrm{mAb}$ (GenScript, Piscataway, NJ) diluted at 1:500 in TBST. After washing in TBST, bound antibody was detected using horseradish peroxidase (HRP)-conjugated goat anti-mouse IgG (Jackson ImmunoResearch Laboratories, Inc., West Grove, PA) diluted 1:1000 in PBS with 0.05\% Tween-20 (PBST), and incubation was continued for $1 \mathrm{~h}$. After extensive washing with TBST, peroxidase activity was detected using 3,3',5,5'-tetramethylbenzidine substrate (KPL Inc., Gaithersburg, MD). 


\section{Propagation and titration of OURT88/3}

The ASFV OURT88/3 strain [15] was propagated and titrated on primary cultures of porcine alveolar macrophages (PAMs) collected by lung lavage from 3- to 5-week old piglets. Prior to infection, PAMs were cultured for two days in RPMI-1640 culture medium (Life Technologies) supplemented with $10 \%$ FBS and 1x antibiotics-antimycotic (Gibco), in a $37{ }^{\circ} \mathrm{C} 5 \% \mathrm{CO}_{2}$ incubator. Two days postinfection, virus-positive cells were detected by indirect immunofluorescence assay (IFA). Briefly, PAMs cultured on 96-well plates were washed with phosphate-buffered saline (PBS) and fixed with $4 \%$ paraformaldehyde, then washed and permeabilized with $0.01 \%$ Triton X-100 (Sigma-Aldrich). Primary antibody consisting of anti-p30 mouse ascites was incubated for $30 \mathrm{~min}$ at $37^{\circ} \mathrm{C}$, followed by washes and 30 min at $37{ }^{\circ} \mathrm{C}$ incubation with IgG goat anti-mouse Alexa Fluor 488 (Life Technologies) secondary antibody. After washing, cell nuclei were counterstained with DAPI (Life Technologies) to differentiate positive cells from debris. The $\log _{10}$ of the $50 \%$ tissue culture infectious dose $\left(\mathrm{TCID}_{50}\right)$ was calculated using the Spearman-Karber method [20].

\section{Animal experiments}

Groups of ten 4-week-old pigs were immunized intramuscularly (IM) with $2 \mathrm{ml}$ containing 2.0-4.5 × 10 $0^{7} \mathrm{RPs}$ of RP-30, RP-54, RP-72 or RP-sHA-p72. Pigs were immunized at three-week intervals at the same dose, and the experiment was terminated four weeks after the third immunization. Pigs were bled before the first immunization and weekly thereafter. Pen-based oral fluid samples were collected from each group daily for 5 days each week. Oral fluid was collected from each group of pigs as described by Prickett et al. [21] and stored at $-20{ }^{\circ} \mathrm{C}$.

For the prime-boost experiment, ten 3 week-old Large White $\times$ Landrace pigs were primed by two immunizations with RP-30 following the same protocol described above. Six pigs were included as non-immunized controls. One week following the second RP-30 immunization, all sixteen pigs were inoculated by IM injection with $10^{4} \mathrm{TCID}_{50}$ of ASFV OURT88/3. Pigs were intermingled throughout the study. Daily temperatures and clinical signs including attitude, body condition, lameness, nervous signs, respiratory signs, fecal quality, and eye and skin conditions were recorded. Each category was scored on a scale from 0 to 3 : 0 being normal, 3 being severe. Elevated temperature $40{ }^{\circ} \mathrm{C}$ $\left(>104{ }^{\circ} \mathrm{F}\right)$ added a point to the overall score. Serum and oral fluid were collected at $0,3,7,10,14,17,20$ and 21 days post-inoculation (DPI) with OURT88/3 and stored at $-80{ }^{\circ} \mathrm{C}$. The study was terminated $21 \mathrm{DPI}$ with OURT88/3; some animals were euthanized at earlier time points for animal welfare reasons. Animals were humanely euthanized by pentobarbital overdose following the American Veterinary Medical Association guidelines for the euthanasia of animals, and all efforts were made to minimize suffering.

\section{Detection of ASFV infection}

Although whole blood is ideally used to test for viremia, serum was obtained from all pigs at all collection times and therefore used for these experiments. Total RNA/DNA was extracted from sera and oral fluid samples collected after immunization with OURT88/3 using a MagMAX ${ }^{\text {TM}}-96$ Viral RNA Isolation Kit according to the manufacturer's protocol (ThermoFisher Scientific, Waltham, Massachusetts, USA). Negative extraction controls were represented by non-infectious samples collected at $0 \mathrm{DPI}$, and positive extraction controls included 10-fold dilutions of OURT 88/3 virus stock. Briefly, $50 \mu \mathrm{l}$ of serum samples were combined with $20 \mu \mathrm{l}$ of bead mix (containing lysis/binding solution, carrier RNA, and $100 \%$ isopropanol) in a U-bottom 96-well plate provided by the kit, and $130 \mu \mathrm{l}$ of lysis/binding solution was added per well. The beads were captured on a magnetic stand and washed twice each with wash solutions 1 and 2 . The final elution volume was $50 \mu \mathrm{l}$ with the provided buffer. The extracted samples and controls were stored at $-80{ }^{\circ} \mathrm{C}$ until analysis by PCR.

The serum and oral fluid samples were tested using an in-house PCR assay based on primers and a probe described by King et al. to amplify a conserved region of p72 [22]. The primers/probe mixture was commercially synthesized using PrimeTime ${ }^{\circledR}$ Mini qPCR Assay (IDT Technologies). The 20- $\mu$ l-volume PCR reaction consisted of $10 \mu \mathrm{l}$ of $2 \mathrm{x}$ iTaq Universal Probes Supermix (Bio-Rad), $1 \mu$ l of $1 \mathrm{X}$ PrimeTime Mini ( $500 \mathrm{nM}$ primers and $250 \mathrm{nM}$ probe), $4 \mu \mathrm{l}$ of nuclease-free water, and $5 \mu$ of sample DNA. Quantitative PCR (qPCR) was conducted using the CFX96 ${ }^{\mathrm{TM}}$ Real-Time System (Bio-Rad) using the following cycling conditions: 1 cycle at $95^{\circ} \mathrm{C}$ for $15 \mathrm{~min}$, followed by 45 cycles of $95^{\circ} \mathrm{C}$ for $15 \mathrm{~s}$ and $60^{\circ} \mathrm{C}$ for $60 \mathrm{~s}$. A signal with a $\mathrm{Ct}$ value $<40$ was considered positive.

Serum samples that tested weakly positive by PCR were further tested by virus isolation. Virus isolation was performed by two passages of serum diluted 1:10 on PAMs. Infectious virus was detected by IFA following the protocol described above.

\section{Expression of recombinant ASFV proteins in E. coli}

The ASFV nucleic acid sequences were codon optimized for expression in E. coli and synthesized by GenScript (GenScript, Piscataway, NJ). The p30, p54, and p72 whole proteins, and 10 overlapping fragments of p30 were subcloned in frame into pHUE [23] and used to transform One Shot BL21 (DE3) cells (Invitrogen, Grand Island, NY). For 
protein production, bacteria were grown overnight in LB broth with $10 \mu \mathrm{g}$ of ampicillin per ml. Two milliliters of overnight culture was added to $100 \mathrm{ml}$ of LB broth and incubated at $37{ }^{\circ} \mathrm{C}$ with shaking. Once the optical density was between 0.4 and 0.6 , protein expression was induced by adding $1 \mathrm{ml}$ of $0.1 \mathrm{M}$ isopropyl $\beta$-D-1-thiogalactopyranoside, and incubation was continued for $4 \mathrm{~h}$. Bacteria were pelleted by centrifugation at $4000 \times g$ for $10 \mathrm{~min}$ and stored at $-20{ }^{\circ} \mathrm{C}$. Proteins were purified from the bacterial pellet using a PrepEase His-Tagged Protein Purification Kit-High Yield (USB Corporation, Cleveland, $\mathrm{OH}$ ), following the manufacturer's protocol for denaturing conditions - p30, p54, p72 whole proteins, $\mathrm{p} 30(1-100), \mathrm{p} 30(50-150)$ - or for native conditions - p30(61-110), p30(111-160), p30(91130), p30(143-182), p30(161-204), p30(101-204). Two p30 fragments, $\mathrm{p} 30(24-60)$ and $\mathrm{p} 30(40-80)$, were purified using a modified protocol with $0.3 \%$ Sarkosyl/0.5M CAPS. The purity of each protein was assessed by SDS PAGE.

\section{RP immunization experiment: detection of antigen-specific antibodies}

Immunoblotting was performed by transferring proteins from SDS PAGE gels to a PVDF membrane (Amersham, GE Healthcare Life Sciences, Pittsburg, PA) using a Mini Trans-Blot Electrophoretic Transfer Cell (Bio-Rad, Hercules, CA) following the manufacturer's instructions. The membrane was incubated overnight at $+4{ }^{\circ} \mathrm{C}$ in blocking buffer containing 5\% NFDM in PBS with $0.1 \%$ Tween-20, followed by three washes with PBST. Mouse anti-penta-His mAb (QIAGEN, Valencia, CA), diluted 1:1000 in blocking buffer, was added to the membrane and incubated for $2 \mathrm{~h}$ at room temperature with shaking. After three washes with PBST, HRP-conjugated goat anti-mouse IgG (ICN Biomedicals Inc., Aurora, OH), diluted 1:500 in blocking buffer, was added to the membrane and incubated for $1 \mathrm{~h}$ at room temperature with shaking. Peroxidase activity was detected with 4-chloro-1-naphtol/3,3'-diaminobenzidine, tetrahydrochloride substrate (Thermo Fisher Scientific Inc., Waltham, MA). When sera from pigs were used as primary antibody, the sera were diluted 1:300 in blocking buffer. The secondary antibody, HRP-conjugated goat anti-swine IgG (Accurate Chemical and Scientific Corporation, Westbury, NY), was diluted 1:3000 in blocking buffer.

ELISA was performed for detection of antigen-specific antibody by adjusting recombinant protein concentrations to $4 \mu \mathrm{g} / \mathrm{ml}$ in carbonated coating buffer $\left(50 \mathrm{mM} \mathrm{Na}_{2} \mathrm{CO}_{3}\right.$, $50 \mathrm{mM} \mathrm{NaHCO}_{3}, \mathrm{pH}$ 9.6) and coating flat-bottom polystyrene ELISA plates (Costar, Corning Inc., NY) with $100 \mu \mathrm{l}$ per well. Wells were blocked for $1-2 \mathrm{~h}$ at $37{ }^{\circ} \mathrm{C}$ with $10 \%$ $(\mathrm{v} / \mathrm{v})$ goat serum in PBS (PBS-GS). After three washes with PBST, $100 \mu \mathrm{l}$ of serum diluted 1:320 in PBS-GS was added to each well and incubated for $1 \mathrm{~h}$ at $37^{\circ} \mathrm{C}$. Modifications for oral fluid samples included $200 \mu \mathrm{l}$ of a 1:2 dilution in PBSGS and incubation overnight at $+4{ }^{\circ} \mathrm{C}$. After three washes with PBST, HRP-conjugated goat anti-swine IgG secondary antibody (Accurate Chemical and Scientific Corporation, Westbury, NY), diluted 1:2000 in PBS-GS, was added to each well, and the plate was incubated for $1 \mathrm{~h}$ at $37^{\circ} \mathrm{C}$, followed by three washes with PBST. Peroxidase activity was detected by adding $100 \mu \mathrm{l}$ of 2,2'-azino-di (3-ethylbenzthiazoline-6-sulfonate) (KPL Inc., Gaithersburg, MD) to each well. After a 20-min incubation at room temperature, the reaction was stopped by adding $1 \%$ SDS (w/v) in double-distilled water. Absorbance was measured using a FLUOstar Omega microplate reader (BMG Labtech, Cary, $\mathrm{NC})$. Results are reported as the absorbance at 405-650 nm.

\section{Confocal microscopy}

Vero cells were cultured in growth medium on glass coverslips in a 24 -well plate until approximately $70 \%$ confluent. Cells were infected with ASFV strain BA71V at an MOI of 0.001 . After 1 hour, the inoculum was removed and replaced with EMEM, 2\% FBS, glutamine, HEPES and antibiotics. At 4, 8, 18 and 24 hours postinfection (hpi), the medium was removed and the monolayers were fixed with $4 \%$ paraformaldehyde for 40 minutes at room temperature, washed three times with PBS, and blocked for 10 minutes with blocking buffer (5\% BSA in PBS). Anti-p30 serum, taken from a single pig immunized with RP-30, was diluted 1:400 in blocking buffer and added to the cells. After a 1-hour incubation at room temperature, the slides were washed three times with PBS. Anti-swine FITC secondary antibody (Jackson Laboratories, Bar Harbor, ME), diluted 1:200 in blocking buffer, was then added, and the incubation continued for 1 hour. After washing, cells were counterstained with 4', 6' diamino-2-phenylindole-2 $\mathrm{HCl}$ (Sigma-Aldrich, St. Louis, MO) for 10 minutes at room temperature, then washed twice with sterile water. Coverslips were mounted on microscope slides and visualized using a Leica SP5 confocal microscope.

\section{Prime-boost experiment: indirect ELISA for anti-p30 antibodies and epitope mapping}

The ELISA procedure for determining anti-p30 antibody reactivity was performed as described above with the following modifications: a positive control serum from a pig at 21 DPI with OURT88/3 and one negative control serum from before vaccination were added to each ELISA test plate. The positive control was added in triplicate with twofold serial dilutions starting at 1:200 in order to determine the dilution corresponding to the half-maximal absorbance. The positive control mean was calculated for each plate at the half-maximal dilution of 1:6400, with 
plate-to-plate variation consisting of 0.11 standard deviation (SD) in absorbance. The negative control and individual pig serum samples from $0,3,7,10,14,17,21$ DPI were tested at a single dilution of 1:400. The absorbance of each sample was calculated as $\left(\mathrm{A}_{405}-\mathrm{A}_{650}\right)$ of the sample divided by the mean of the half-maximal absorbance $\left(\mathrm{A}_{405}-\mathrm{A}_{650}\right)$ of the positive control serum. The normalized negative control mean and standard deviation were calculated and used to determine the cutoff of the assay. The cutoff of the assay was set at 0.4 , which was the highest value of the negative control mean $+3 *$ SD that was obtained between plates (Fig. 5a). In order to determine p30 antibody titers from sera, samples from 0 and 17 DPI were diluted twofold starting at 1:200. Antibody titers were calculated as the reciprocal of the sample dilution at half of the maximal absorbance (Fig. 5b).

The ELISA procedure for $\mathrm{p} 30$ epitope mapping was performed by coating plates with nine overlapping fragments of $\mathrm{p} 30$. The whole $\mathrm{p} 30$ protein was used as a positive control. Serum samples from experimentally vaccinated and ASFV-infected pigs were used at a 1:400 dilution. As described above, a negative and a positive control serum were included in each plate. Absorbance values were calculated as the mean $\mathrm{A}_{405}-\mathrm{A}_{650}$ value for the sample minus the mean $\mathrm{A}_{405}-\mathrm{A}_{650}$ value for the negative control.

\section{Virus neutralization assay}

Sera were heat-inactivated at $56{ }^{\circ} \mathrm{C}$ for 30 minutes and then diluted 1:10 in complete RPMI media. Each sample was assayed in triplicate and included a virus-only control (no serum) on each assay plate. One hundred microliters of the serum dilution was mixed with $100 \mu \mathrm{l}$ of ASFV OURT $88 / 3$ strain at $500 \mathrm{TCID}_{50} / \mathrm{ml}$. The mixture of virus and serum was incubated for $1 \mathrm{~h}$ at $37^{\circ} \mathrm{C}$ and subsequently added to PAMs cultured in a 96-well plate for two days prior to the assay. The plate was incubated for $1 \mathrm{~h}$ at $37^{\circ} \mathrm{C}$, in $5 \% \mathrm{CO}_{2}$, and the inoculum was then replaced with fresh medium. PAMs were incubated for 3-4 days at $37{ }^{\circ} \mathrm{C}$ in $5 \% \mathrm{CO}_{2}$, and IFA was then performed. Using the software of the EVOS FL Auto microscope (ThermoFisher Scientific), each well was divided into 53 fields, and 10 were selected to determine the total number of cells and fluorescent positive cells. For each sample, the average percentage of fluorescent cells out of the total number of cells for three replicate wells was determined. Because no positive neutralizing serum for use as a standard control was available at the time of assay, neutralization was based on the virus-only control. The $\%$ neutralization was calculated using the following formula: ((1- (Mean \% positive cells of sample serum / Mean \% positive cells of virus only control))*100).

\section{Results}

\section{Expression of ASFV-alphavirus constructs in Vero cells}

Expression and titration of RPs was assessed by infection of Vero cells and subsequently staining for the presence of VEEV nsp2. Positive nsp2 staining was observed for all constructs (Fig. 1a), demonstrating that RP-30, RP-54 and RP-72 were able to undergo one cycle of replication for expression of the respective target antigens. The modified construct, RP-sHA-p72, was also positive for nsp2 expression (data not shown). ASFV protein expression in Vero cells was assessed by immunoblotting immobilized proteins with an anti-6xHis mAb. As shown in Fig. 1b, all recombinant proteins were detected and migrated at the predicted molecular weights. Some background was observed, which could be due to the anti-his tag antibody reacting with other cell lysate proteins. Even though cells were infected with the same number of virus particles, the results revealed widely different levels of ASFV protein expression. The highest level of expression was obtained for the RP-30 construct, while RP-54, RP-72 and RPsHA-72 were expressed at much lower levels compared to RP-30.

\section{Antibody response against bacteria-expressed proteins after immunization with RPs}

Pigs were immunized three times with each RP construct, and serum and oral fluids were collected throughout the study. The results for antibody reactivity in serum and oral fluid samples are presented in Fig. 2. Antibody activity against recombinant $\mathrm{p} 30$ was first detected in all pigs at one week after the second immunization on day 28 (Fig. 2a). Spikes in mean immunoreactivity to p30 in sera were observed following the second and third RP-30 immunizations, at 35 and 56 days (Fig. 2a). Beginning at day 27, p30 reactivity was detected in the oral fluid samples. Overall, the results for the oral fluid samples followed a pattern similar to the anti-p30 activity in serum. Only three pigs immunized with RP-54 produced detectable amounts of anti-p54 binding activity in serum (Fig. 2b). Elevated anti-p54 activity was first detected on day 55, two weeks after the third immunization. A single pig showed higher-than-background levels of anti-p54 activity, beginning at 14 days. Absorbance values for anti-p54 activity in the oral fluid samples remained at background levels for the entire study period. Serum samples from pigs immunized with RP-72 remained at background levels over the entire study period (data not shown). Similar 
a

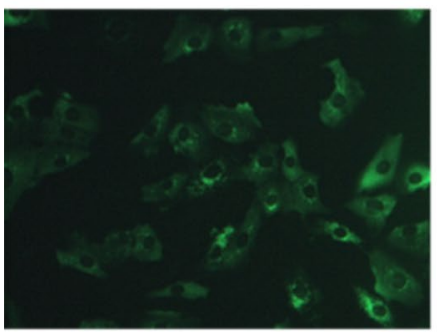

RP-30

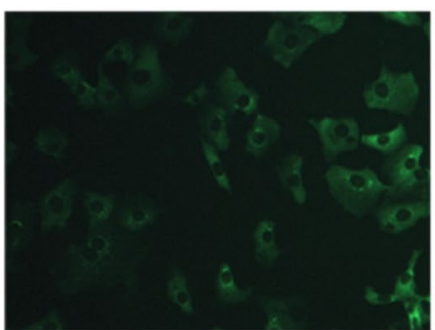

RP-72

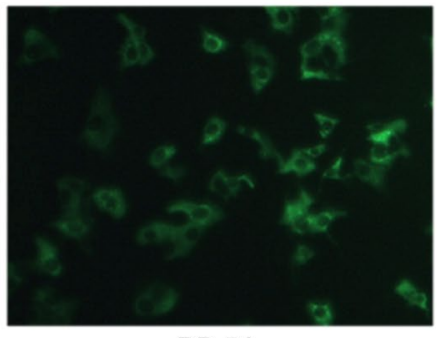

RP-54

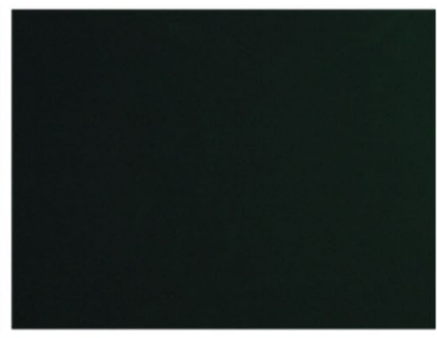

Mock infected

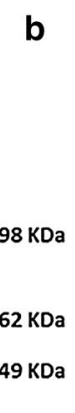

b Std RP- Control SHA-72 Lysate

$38 \mathrm{KDa}$

$28 \mathrm{KDa}$

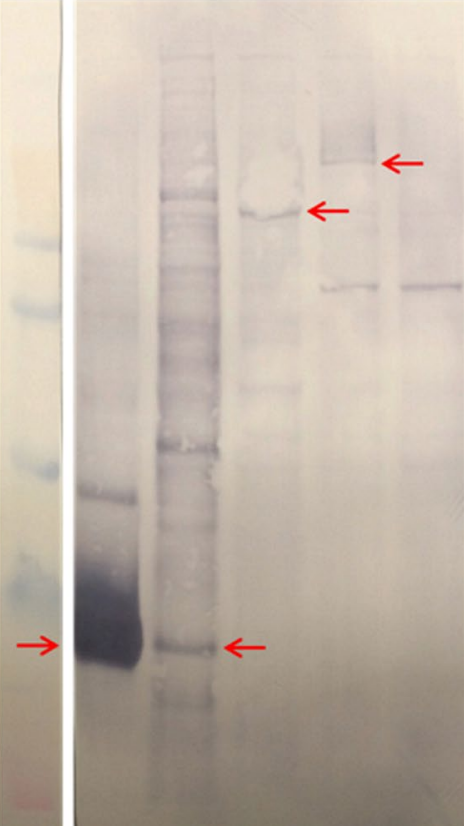

Fig. 1 Expression of alphavirus and recombinant RP ASFV proteins in Vero cells. (a) Immunofluorescence staining of Vero cells infected with RP ASFV protein constructs, using anti-VEEV-nsp2. (b) Western blot of RP-infected cells stained with anti-His mAb. For compari-

to Argilaguet et al. [8], we attempted to increase the immunogenicity of p72 by adding the HA domain from the ASFV CD2-like protein. A 197-amino-acid extracellular HA region, including the signal peptide sequence, was added to the $\mathrm{N}$-terminal end of the $\mathrm{p} 72$ construct to produce RP-sHA-p72. The additional polypeptide did not increase the expression of $\mathrm{p} 72$ in infected Vero cells (see Fig. 1b). Two pigs, beginning at day 49, or one week after the third immunization with RP-sHA-72, showed measureable anti-p72 activity (Fig. 2c). Oral fluid samples also showed a measureable increase in anti-p72 reactivity at around the same time.

\section{Recognition of p30 expressed in ASFV-infected Vero cells by immune sera}

The specificity of sera from RP-30-immunized pigs was further investigated by following the localization of anti-p30 staining in Vero cells infected with ASFV. Serum from a single pig at 55 days after the first immunization was tested. The confocal microscopy results, presented in Fig. 3, showed that antibody staining was localized in the cytoplasm at $4 \mathrm{~h}$ after infection. By $18 \mathrm{~h}$, there was further accumulation of anti-p30 staining in the cytoplasm and the nucleus (Fig. 3a). By $24 \mathrm{~h}$, fluorescence was localized to structures that resembled virus assembly sites in the perinuclear region of son, Vero cells were infected with the same number of replicon particles, and the same amount of total protein was loaded into each well. Red arrows identify the location of the predicted size for each protein. Protein standards (std) are indicated on the left

infected cells (Fig. 3b). This pattern of staining is similar to the intracellular localization of p30 reported previously [24]. Together, the results indicate that the RP-30-immunized pigs produced antibodies that recognized both recombinant and native forms of $\mathrm{p} 30$.

\section{Prime-boost experiment}

Pigs were primed with two RP-30 immunizations at a fourweek interval and then boosted with OURT88/3 one week later (RP-30 + OURT88/3). Pigs that were not immunized with RP-30 but infected with OURT88/3 were also included (OURT88/3 only). Following infection with OURT88/3, slightly elevated rectal temperatures and low clinical scores were observed among the RP-30 + OURT $88 / 3$ primeboosted and OURT88/3-only groups of pigs. The most common clinical signs were pyrexia, joint swelling, and lameness. Adverse reactions such as pyrexia and arthritis associated with OURT88/3 have been reported previously $[16,18]$. Two control pigs (\#48 and \#75) developed vesicular lesions on the skin that deteriorated into open sores. One pig each from the OURT88/3-only group (\#75) and from the RP-30 + OURT88/3 group (\#190) was euthanized two days prior to the study termination date for animal-welfare reasons. 

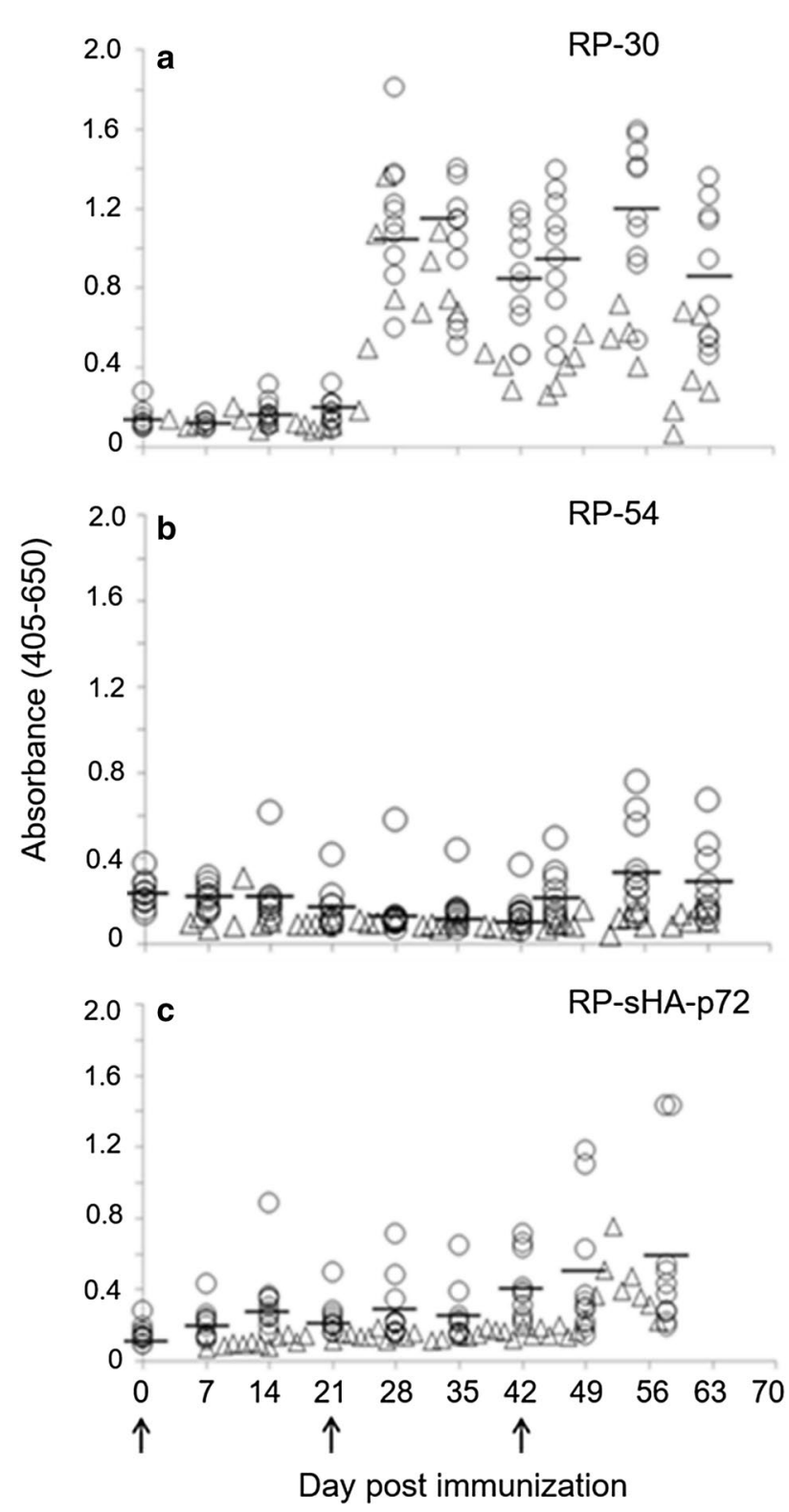

Fig. 2 Antibody responses of pigs immunized with RP-30, RP-54, RP-sHA-p72. Serum samples were diluted 1:320, and oral fluid was diluted 1:2. Panel a shows the anti-p30 activity of RP-30-immunized pigs. Panels $b$ and $c$ show anti-p54 and anti-p72 activity of RP-54and RP-sHA-p72-immunized pigs, respectively. Open circles show $\mathrm{A}_{405}-\mathrm{A}_{650}$ values for individual pigs. The arrows identify the immunization days and horizontal bars show the mean $\mathrm{A}_{405}-\mathrm{A}_{650}$ values for serum. The triangles show the $\mathrm{A}_{405}-\mathrm{A}_{650}$ values for the oral fluid samples

The results for ASFV quantitative PCR, shown in Fig. 4, identified three pigs each from the RP-30 + OURT $88 / 3$ prime-boosted group and the OURT88/3-only group as weakly positive for the presence of ASFV nucleic acid $(33<\mathrm{Ct}<40)$ at 7,14 and $17 \mathrm{DPI}$. However, these sera tested negative for ASFV by virus isolation on macrophages (data not shown). Serology was performed to test antibody binding activity against the whole recombinant $\mathrm{p} 30$ protein. The results presented in Fig. 5a showed high immunoreactivity to $\mathrm{p} 30$ from 0 to 21 DPI among the RP-30 + OURT88/3 prime-boosted pigs and seroconversion of some pigs in the OURT88/3-only group by 7 DPI. Although not considered statistically significant, an increase in mean p30 antibody titer was observed after boost with OURT88/3 (Fig. 5b). However, there was no difference in $\mathrm{p} 30$ antibody titers at 17 DPI between the RP-30 + OURT88/3 prime-boosted pigs, and the group that received OURT88/3 only (Fig. $5 b$ ).

\section{Recognition of $p 30$ epitopes}

To identify potential p30 epitopes, sera from OURT88/3infected pigs collected at 0 and 17 DPI were tested by ELISA against overlapping fragments of p30 (see Fig. 6a). Sera were divided into three groups: sera collected at 0 DPI from pigs primed two times with RP-30 only, 17 DPI sera from pigs infected with OURT88/3 only, and 17 DPI sera from RP-30 + OURT88/3 prime-boosted pigs. As shown in Fig. 6, sera from all groups reacted with the whole $\mathrm{p} 30$ recombinant protein consisting of amino acid residues 1-204. Select sera were also assayed for the presence of neutralizing activity. Pigs immunized with RP-30 only possessed low amounts of activity, whereas, sera from the OURT88/3-only group and RP-30 + OURT88/3 prime-boosted pigs possessed detectable amounts of neutralizing activity (see Fig. 7).

Antibody activity was readily detected when using p30(50-150) and p30(101-204) polypeptides as target antigens (see Fig. 6b). The fragments covered the overlapping region $\mathrm{p} 30(101-150)$. Fragment $\mathrm{p} 30(1-100)$ possessed the least reactivity. The $\mathrm{p} 30(1-100)$ overlapped with the $\mathrm{p} 30(50-$ 150) polypeptide covering p30(50-100). Therefore, the immunodominant region is likely to be located in the region of amino acids 101-150 of p30. The greatest effect of RP-30 priming was found in the recognition of the p30(1-100) fragment. As shown in Fig. 6b, antibody activity was significantly higher in the RP-30 + OURT $88 / 3$ prime-boosted pigs when compared to either the RP-30-only or OURT88/3-only group. Another difference was found in the number of pigs in each group that recognized the p30(1-100) fragment. For RP-30 only and OURT88/3 only, 8 of 10 and 1 of 6 pigs recognized the $\mathrm{p} 30(1-100)$ polypeptide, respectively, whereas all 10 pigs in the RP-30 + OURT88/3 prime-boosted group recognized the same fragment (see Fig. 6c). Taken together, these results show that priming with RP-30 prior to infection with OURT $88 / 3$ expanded the recognition of minor epitopes located near the N-terminal end of p30.

Finer mapping was conducted using a set of overlapping oligopeptides that covered amino acids 24 to 204 of p30. The greatest amount of activity was found in the recognition of two overlapping oligopeptides, p30(91-130) and p30(111160) (see Fig. 8a). Interestingly, reactivity was greatest for 
Fig. 3 Immune serum recognizes native $\mathrm{p} 30$ expressed by (DAPI) and anti-p30 (FITC) staining at $18 \mathrm{~h}$ after infection. (b) Merged photomicrograph at $24 \mathrm{~h}$ after infection showing the presence of a virus assembly site, enlarged in the inset. Dotof virus assembly sites. White arrows show the accumulation of p30 in the nucleus infected Vero cells. (a) Confocal photomicrograph showing DNA ted circles show the location
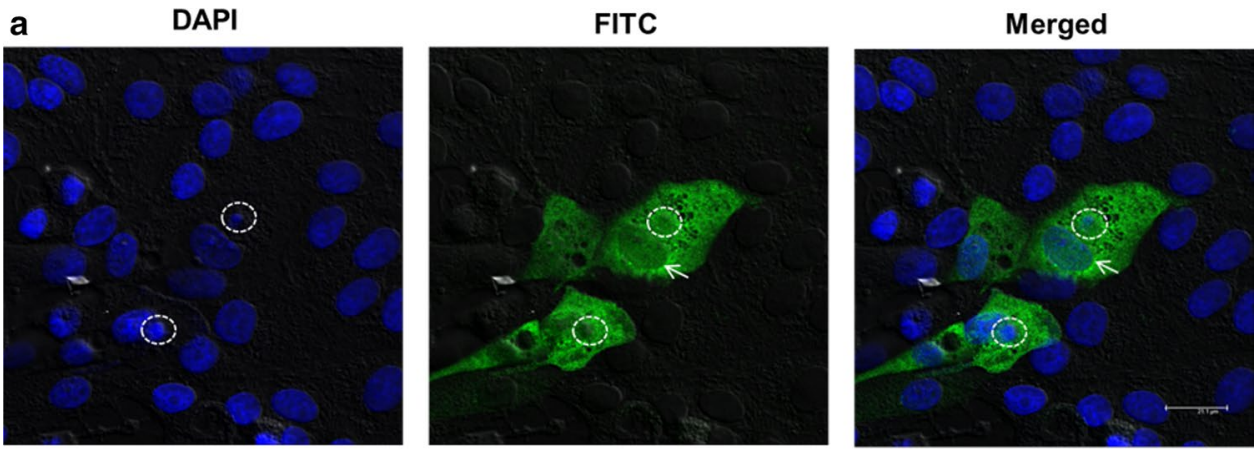

b

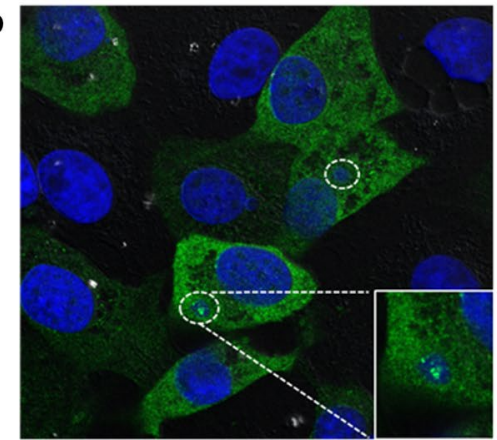

further, the data suggest that a short linear epitope is located between amino acids 111 and 130 .

Although no difference in p30 antibody levels was observed between the RP-30 + OURT88/3 prime-boosted and OURT88/3-only groups of pigs (Fig 5b), the main effect of RP-30 priming with the OURT88/3 boost was found in the recognition of the p30(61-110) oligopeptide. A significant increase in reactivity was observed for the RP-30 + OURT88/3 group of pigs compared to RP-30-only group, as shown in Fig. 8a. In addition, 9 of 10 pigs in the RP-30+ OURT88/3 prime-boosted group recognized p30(61-110), compared to 1 of 10 and 3 of 6 pigs for the RP-30-only and OURT88/3-only group, respectively (Fig. 8b). This oligopeptide is located in the vicinity of the 50-100 peptide sequence covered by the overlap between the p30(1-100) and p30(50-150) polypeptides (see Fig. 6b).

ASFV p72 qPCR on serum collected at the indicated postinfection (DPI) with OURT88/3. The floating open bars illustrate the range of $\mathrm{Ct}$ values with the group means represented by the solid line. The dotted line shows the assay cutoff. $\mathrm{Ct}$ values $<40$ (above the dotted line) were considered ASFV positive, and $\mathrm{Ct}>40$ (below the dotted line) were considered negative

the OURT88/3-only and RP-30 + OURT88/3 prime-boosted pigs. The reactive oligopeptides are found in the same region covered by the $\mathrm{p} 30$ (50-150) and $\mathrm{p} 30$ (101-204) polypeptides (see Fig. 6b). The p30(91-130) and p30(111-160) oligopeptides contain the 20-amino-acid overlapping region (111130) of the sequence ETNECTSSFETLFEQEPSSE. The 111-130 peptide sequence is located within the 101-150 overlap covered by polypeptides $\mathrm{p} 30(50-150)$ and $\mathrm{p} 30(101-$ 204) shown in Fig. 6b. Although it was not investigated

\section{Discussion}

Prime-boost strategies involving two different vaccine platforms create an opportunity to produce a synergistic effect when compared to either vaccine alone. Alphavirus-vectored vaccines have been established as safe and effective for use against other viral swine diseases; however, this approach has not yet been explored for use against ASF. In this study, alphavirus-expressed ASFV immunogenic proteins p30, $\mathrm{p} 54$, and $\mathrm{p} 72$ were tested as potential priming antigens in a prime-boost approach with an attenuated live virus vaccine candidate, OURT88/3. We identified a correlation between 

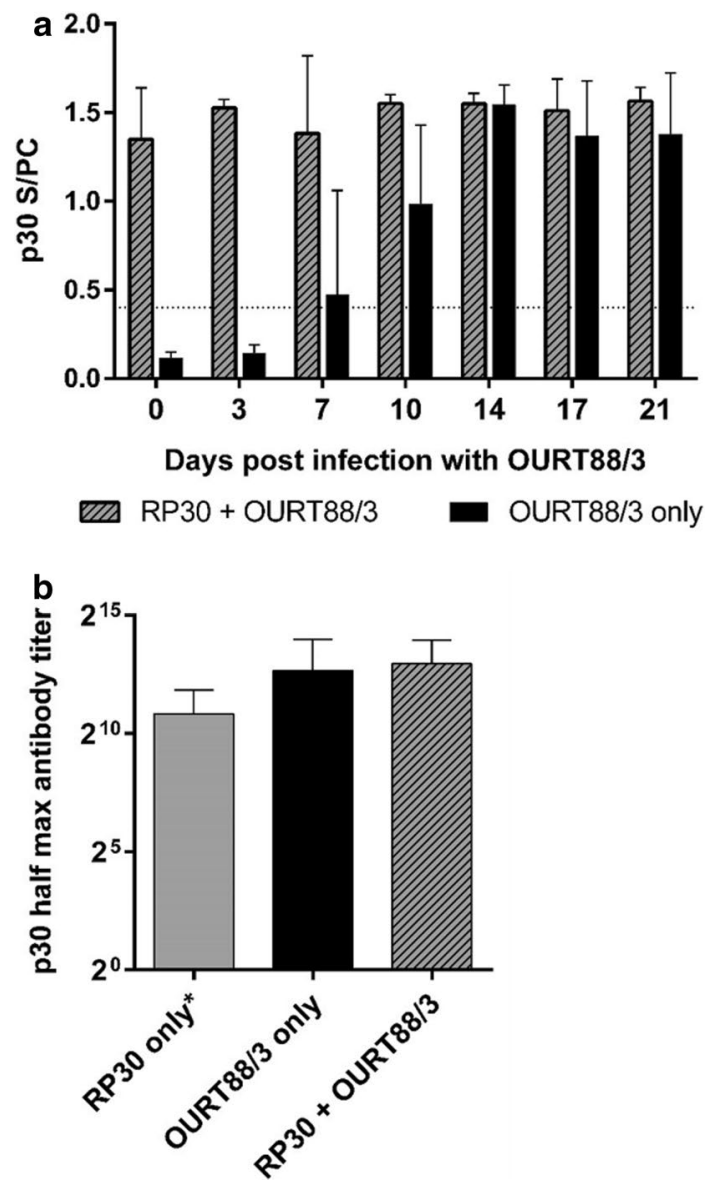

Fig. 5 Anti-p30 antibody response at the indicated days postinfection with OURT88/3. (a) Results of whole p30 recombinant protein ELISA with pig serum at 1:400 are shown as sample (S)-to-positive control (PC) ratios with standard deviation as described in Materials and methods. The cutoff for the assay was 0.4 , as indicated by the dotted line. (b) Serum p30 antibody titers were calculated as the reciprocal of the dilution factor for half-maximal absorbance of the sample. RP30 + OURT88/3 and OURT88/3-only groups represent sera collected at $17 \mathrm{DPI}$. The RP30-only group represents sera collected at 0 DPI from pigs primed two times with RP30 but not yet boosted with OURT88/3. No statistical significance was found between the groups when assessed by one-way ANOVA in GraphPad Prism version 6

protein expression in vitro and immunogenicity in vivo, which allows for the prediction of RP constructs that are most likely to be immunogenic. Priming pigs with RP-30 induced specific antibodies, and boosting with OURT88/3 resulted in increased p30 antibody levels, although there was no significant difference observed between mean p30 antibody titers of the RP-30 + OURT $88 / 3$ group of pigs and the pigs that received OURT88/3 only (see Fig. 5b). Nonetheless, the results suggest that combining RP-30 and OURT $88 / 3$ in a prime-boost approach could enhance recognition of minor epitopes, such as the region within the vicinity of the 61-110 peptide sequence of $\mathrm{p} 30$. This was a

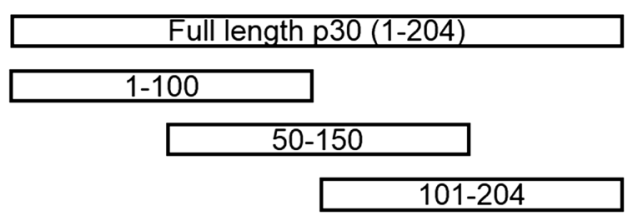

b

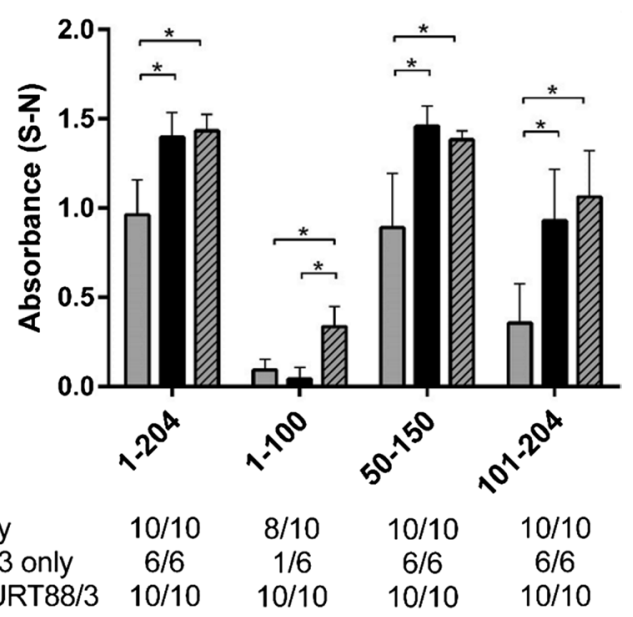

Fig. 6 Epitope mapping of p30. (a) Pig sera were tested at 1:400 by ELISA against p30 whole protein or overlapping polypeptides; corresponding amino acids are indicated. The RP30 + OURT88/3 and OURT88/3-only groups represent sera collected at 17 DPI. The RP30-only group represents sera collected at 0 DPI from pigs primed two times with RP30 but not yet boosted with OURT88/3. Absorbance values are shown as sample $(\mathrm{S})$ minus negative control mean $(\mathrm{N})$ for each fragment. Significant differences between group means for each fragment $(*)$ were calculated by two-way ANOVA with Tukey's multiple comparison test with a 95\% confidence interval in GraphPad Prism version 6. (b) The number of reactive over total pigs from each group is shown for each oligopeptide. Values $\geq 0.05$ indicate reactivity

reflected by a significant increase in $\mathrm{p} 30$ reactivity as well as by the number of pigs that reacted to this region (see Fig. 8).

In addition, the results of $\mathrm{p} 30$ epitope mapping indicated that the immunodominant region resides in a short oligopeptide covered by residues 111-130. We predict this to be shared across strains given the highly conserved nature of p30 among diverse field isolates of ASFV [25]. P30 is a phosphoprotein, and the 111-130 amino acid region is predicted to contain four of the 17 total serine phosphorylation sites at residues $117,118,128$ and 129 [26]. The 111-130 peptide sequence also overlaps a putative $\mathrm{N}$-glycosylation site at residues 110-112 [26], yet the function of these posttranslational modification sites on $\mathrm{p} 30$ has not been determined, and it is unclear whether p30 is actually glycosylated. The results presented here and identification of epitopes on other viral antigens will be valuable for producing an ASF vaccine and diagnostic development and refinement.

The role of p30 antibodies and the effect of expansion of immunogenic epitopes on protection from virulent challenge 


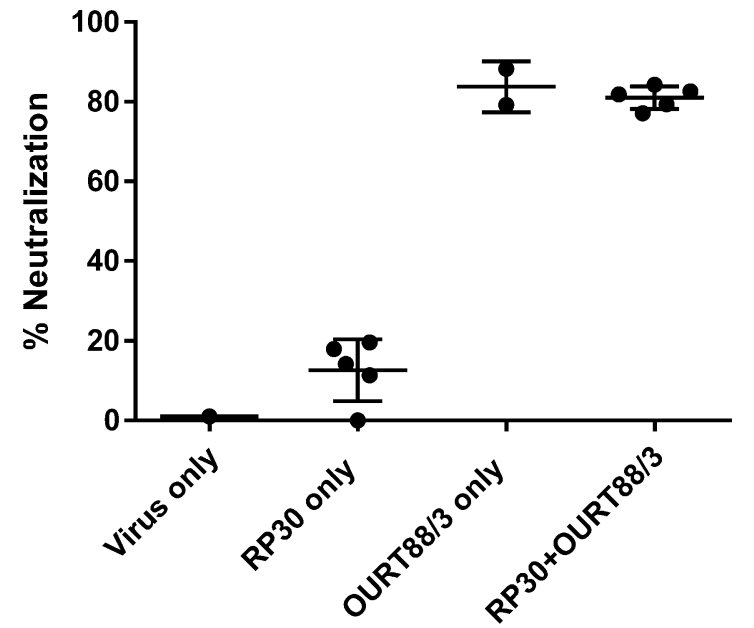

Fig. 7 Virus neutralizing activity. A total of 12 pig sera were evaluated: five sera from the RP-30-only group of pigs collected at 0 DPI, 17 DPI sera from two pigs that received OURT88/3 only, and five 17 DPI sera from RP-30 + OURT $88 / 3$ prime-boosted pigs. The virusonly control contained no serum. Sera diluted 1:10 were incubated with $500 \mathrm{TCID}_{50}$ of OURT88/3 virus per ml. The mean percentage of immunofluorescent virus-positive cells was used to calculate percent neutralization based on the virus-only control using the following equation: ((1- (Mean \% positive cells of sample serum / Mean \% positive cells of virus only control) $) * 100$ )

remain to be seen. In general, it is still not clear which ASFV immunogens are important for eliciting a protective response. Furthermore, the role of anti-ASFV neutralizing antibodies in conferring protection against virulent challenge remains controversial. Immunization with p30 alone or in combination with other antigenic ASFV proteins has resulted in various outcomes after virulent challenge, ranging from absence of protection to partial protection [5-7]. Our results indicated that antibodies induced by RP-30 alone were not sufficient for neutralizing virus infection in vitro as compared to infection with OURT88/3 (see Fig. 7), which expresses additional viral neutralizing antigens such as p54 and p72 [5, 27]. It remains unclear what confers protection against ASFV, but delivery of multiple antigens is likely to be required. This may include non-neutralizing targets important for regulating the cell-mediated immune response.

Growing evidence indicates the cell-mediated response plays a critical role in clearing infection [28]. For example, cytotoxic $\mathrm{CD}^{+} \mathrm{T}$ lymphocytes have been indicated to play an important role in protection against ASFV [29]. An advantage of the alphavaccine platform is its ability to stimulate both humoral and cellular immunity. Although cell-mediated responses were not evaluated in this study, the ability of VEEV alphavirus-vectored RPs to induce antigenspecific interferon- $\gamma$-producing cells has been demonstrated in swine for other pathogens such as swine influenza virus [12]. Additional studies are required to know the effect of this prime-boost approach on T-cell-mediated responses. Elucidating how ASFV interacts and affects host immunity will be critical for designing better vaccine formulations, vaccination strategies, and antiviral therapies.

To date, efforts to develop a safe and protective vaccine against ASF have been mostly unsuccessful. Improved protection with attenuated live viruses often comes with adverse side-effects, while subunit or vectored vaccines do not provide the same level of protection. Improvements to attenuated live ASFV vaccines are needed, including expanding cross-protection among multiple viral genotypes and

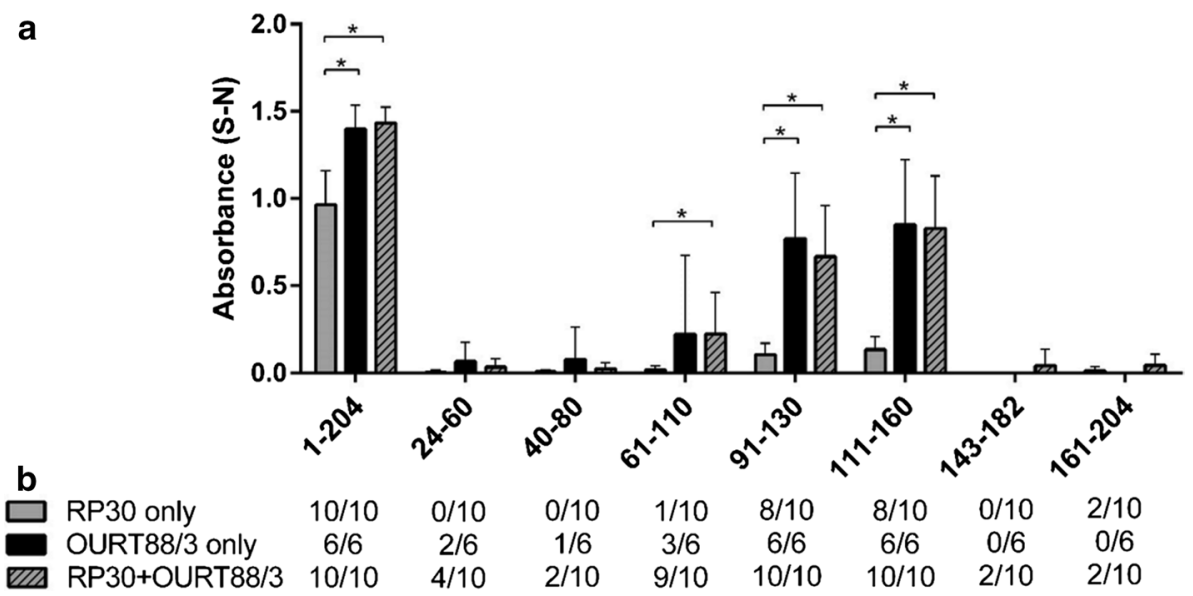

Fig. 8 Fine mapping of p30. (a) Pig sera were tested at 1:400 by ELISA against p30 overlapping oligopeptides; the corresponding amino acids are indicated. The RP30 + OURT88/3 and OURT88/3only groups represent sera collected at 17 DPI. The RP30-only group represents sera collected at 0 DPI from pigs primed two times with RP30 but not yet boosted with OURT88/3. Absorbance values are shown as sample $(\mathrm{S})$ minus negative control mean $(\mathrm{N})$ for each fragment. Significant differences between group means for each fragment (*) were calculated by two-way ANOVA with Tukey's multiple comparison test with a 95\% confidence interval in GraphPad Prism version 6. (b) The number of reactive over total pigs from each group is shown for each oligopeptide. Values $\geq 0.05$ indicate reactivity 
serotypes and reducing the clinical side-effects associated with vaccination. As a proof of concept, we evaluated the delivery and immunogenicity of ASFV antigens using the alphavaccine platform, and furthermore, its incorporation into a prime-boost strategy with an attenuated ASFV strain. While it is clear from other studies that cellular immunity plays an important role in protection against disease, the establishment of specific-antibody responses is critical for effective vaccine development and thus was the focus of analysis in this study. Optimization is clearly important for sufficient expression of different antigens when using a vectored platform, which subsequently affects the magnitude of the antibody response elicited, as observed with the antigens we tested. In order to select the most appropriate antigens, further studies that identify the functional roles of ASFV proteins are critically needed. Our results showed that priming with RP-30 did not appear to affect the response elicited by boosting with OURT88/3. While the ability of RP-30 + OURT88/3 to protect against virulent ASFV challenge was beyond the scope of this study, the results of this work lay the foundation for future efforts towards a safe, effective and cross-protective ASF vaccine. The immunodominant epitope of p30 identified in this study will be beneficial for refined diagnostic and vaccine development.

Acknowledgements We would like to acknowledge Dr. Baker for the contribution of the pHUE expression vector. We also thank Luca Popescu, Vlad Petrovan, Ana Stoian, and the personnel at the Biosecurity Research Institute for technical assistance.

Funding This work was funded by the Kansas National Bio and AgroDefense Facility (NBAF) Transition Fund. Partial support was from the Department of Homeland Security Center of Excellence for Emerging and Zoonotic Animal Diseases (CEEZAD) (Grant no. 2010-ST061AG0001), and Kansas Biosciences Authority/CEEZAD matching funds (Award no. BG2428)

\section{Compliance with ethical standards}

Conflict of interest The authors declare no conflict of interest.

Ethical approval Experiments involving animals and viruses were performed in accordance with the Federation of Animal Science Societies Guide for the Care and Use of Agricultural Animals in Research and Teaching, the USDA Animal Welfare Act and Animal Welfare Regulations, and were approved by the Kansas State University animal care and use committees and institutional biosafety committees. This article does not contain any studies with human participants performed by any of the authors.

\section{References}

1. Alonso C, Borca M, Dixon L, Revilla Y, Rodriguez F, Escribano JM, ICTV Report Consortium (2018) ICTV virus taxonomy profile: Asfarviridae. J Gen Virol 99(5):613-614
2. Tabares E, Martínez J, Martín E, Escribano JM (1983) Proteins specified by African Swine Fever virus. IV. Glycoproteins and phosphoproteins. Arch Virol 77:167-180

3. Escribano JM, Tabares E (1987) Proteins specified by African swine fever virus: V. Identification of immediate early, early and late proteins. Arch Virol 92:221-232

4. Kollnberger SD, Gutierrez-Castañeda B, Foster-Cuevas M, Corteyn A, Parkhouse RM (2002) Identification of the principal serological immunodeterminants of African swine fever virus by screening a virus cDNA library with antibody. J Gen Virol 83:1331-1342

5. Gomez-Puertas P, Rodríguez F, Oviedo JM, Brun A, Alonso C, Escribano JM (1998) The African swine fever virus proteins p54 and p30 are involved in two distinct steps of virus attachment and both contribute to the antibody-mediated protective immune response. Virology 243:461-471

6. Neilan JG, Zsak L, Lu Z, Burrage TG, Kutish GF, Rock DL (2004) Neutralizing antibodies to African swine fever virus proteins p30, p54, and p72 are not sufficient for antibody-mediated protection. Virology 319:337-342

7. Barderas MG, Rodríguez F, Gómez-Puertas P, Avilés M, Beitia $\mathrm{F}$, Alonso $\mathrm{C}$ et al (2001) Antigenic and immunogenic properties of a chimera of two immunodominant African swine fever virus proteins. Arch Virol 146:1681-1691

8. Argilaguet JM, Pérez-Martín E, Nofrarías M, Gallardo C, Accensi F, Lacasta A et al (2012) DNA vaccination partially protects against African swine fever virus lethal challenge in the absence of antibodies. PLoS One 7:e40942

9. Argilaguet JM, Pérez-Martín E, López S, Goethe M, Escribano JM, Giesow K et al (2013) BacMam immunization partially protects pigs against sublethal challenge with African swine fever virus. Antiviral Res 98:61-65

10. Mogler M, Erdman M,Vander Veen R, Harris DLH, Owens G, Kamrud K et al (2009) Replicon particle PRRSV vaccine provides partial protection from challenge. In: Proceedings of the American Association of Swine Veterinarians, 40th annual meeting, pp 367-368

11. Mogler M, Vander Veen R, McVicker J, Russel B, Harris DLH (2010) Vaccination of pigs with PRRVENT or PrrSV-RP recombinant vaccines reduces viremia following heterologous challenge. In: Proceedings of the American Association of Swine Veterinarians, 41st annual meeting, pp 403-404

12. Vander Veen RL, Loynachan AT, Mogler MA, Russell BJ, Harris DLH, Kamrud KI (2012) Safety, immunogenicity, and efficacy of an alphavirus replicon-based swine influenza virus hemagglutinin vaccine. Vaccine 30:1944-1950

13. Vander Veen RL, Mogler MA, Russell BJ, Loynachan AT, Harris DLH, Kamrud KI (2013) Haemagglutinin and nucleoprotein replicon particle vaccination of swine protects against the pandemic H1N1 2009 virus. Vet Rec 173:344

14. Mogler MA, Gander J, Harris DLH (2014) Development of an alphavirus RNA particle vaccine against portice epidemic diarrhea virus. In: Proceedings of the American Association of Swine Veterinarians, 45th annual meeting, pp 63-64

15. Boinas FS, Hutchings GH, Dixon LK, Wilkinson PJ (2004) Characterization of pathogenic and non-pathogenic African swine fever virus isolates from Ornithodoros erraticus inhabiting pig premises in Portugal. J Gen Virol 85:2177-2187

16. King K, Chapman D, Argilaguet JM, Fishbourne E, Hutet E, Cariolet R, Hutchings G, Oura CA, Netherton CL, Moffat K, Taylor G, Le Potier MF, Dixon LK, Takamatsu HH (2011) Protection of European domestic pigs from virulent African isolates of African swine fever virus by experimental immunisation. Vaccine 29:4593-4600

17. Mulumba-Mfumu LK, Goatley LC, Saegerman C, Takamatsu HH, Dixon LK (2016) Immunization of African indigenous pigs 
with attenuated genotype I African swine fever virus OURT88/3 induces protection against challenge with virulent strains of genotype I. Transbound Emerg Dis 63:e323-e327

18. Abrams CC, Goatley L, Fishbourne E, Chapman D, Cooke L, Oura CA, Netherton CL, Takamatsu HH, Dixon LK (2013) Deletion of virulence associated genes from attenuated African swine fever virus isolate OUR T88/3 decreases its ability to protect against challenge with virulent virus. Virology 443:99-105

19. Kamrud KI, Custer M, Dudek JM, Owens G, Alterson KD, Lee JS et al (2007) Alphavirus replicon approach to promoterless analysis of IRES elements. Virology 360:376-387

20. Hierholzer JC, Killington RA (1996) 2-Virus isolation and quantitation A2-Mahy, Brian WJ. In: Kangro HO (ed) Virology methods manual. Academic Press, London, pp 25-46. https://doi. org/10.1016/B978-012465330-6/50003-8 (Prickett J, Simer R)

21. Christopher-Hennings J, Yoon K-J, Evans RB, Zimmerman JJ (2008) Detection of Porcine reproductive and respiratory syndrome virus infection in porcine oral fluid samples: a longitudinal study under experimental conditions. J Vet Diagn Investig 20:156-163

22. King DP, Reid SM, Hutchings GH, Grierson SS, Wilkinson PJ, Dixon LK, Bastos AD, Drew TW (2003) Development of a TaqMan PCR assay with internal amplification control for the detection of African swine fever virus. J Virol Methods 107:53-61

23. Catanzariti A-M, Soboleva TA, Jans DA, Board PG, Baker RT (2004) An efficient system for high-level expression an easy purification of authentic recombinant proteins. Protein Sci 13:1331-1339

24. Hernaez B, Escribano JM, Alonso C (2008) African swine fever virus protein $\mathrm{p} 30$ interaction with heterogeneous nuclear ribonucleoprotein $\mathrm{K}$ (hnRNP-K) during infection. FEBS Lett 582:3275-3280

25. Oviedo JM, Rodriguez F, Gomez-Puertas P, Brun A, Gomez N, Alonso Cand Escribano JM (1997) High level expression of the major antigenic African swine fever virus proteins p54 and p30 in baculovirus and their potential use as diagnostic reagents. J Virol Methods 64:27-35

26. Prados FJ, Vinuela E, Alcami A (1993) Sequence and characterization of the major early phosphoprotein p32 of African swine fever virus. J Virol 67(5):2475-2485

27. Gomez-Puertas P, Rodríguez F, Oviedo JM, Ramiro-Ibáñez F, Ruiz-Gonzalvo F, Alonso C et al (1996) Neutralizing antibodies to different proteins of African swine fever virus inhibit both virus attachment and internalization. J Virol 70:5689-5694

28. Takamatsu HH, Denyer MS, Lacasta A, Stirling CM, Argilaguet JM, Netherton CL, Oura CA, Martins C, Rodriguez F (2013) Cellular immunity in ASFV responses. Virus Res 173:110-121

29. Oura CA, Denyer MS, Takamatsu H, Parkhouse RM (2005) In vivo depletion of $\mathrm{CD} 8+\mathrm{T}$ lymphocytes abrogates protective immunity to African swine fever virus. J Gen Virol 86:2445-2450 